\title{
A FULLY AUTOMATED INCREMENTAL PHOTOGRAMMETRIC PROCESSING DEDICATED FOR COLLABORATIVE REMOTE-COMPUTING WORKFLOW
}

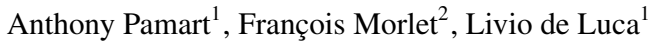 \\ ${ }^{1}$ Modèles et simulations pour l'Architecture et le Patrimoine, UMR 3495 CNRS/MC, Marseille, France \\ ${ }^{2}$ Centre Interdisciplinaire de Conservation et de Restauration du Patrimoine, LABCOM CICRP/MAP, Marseille, France
}

Commission II

KEY WORDS: Close-Range Photogrammetry, Structure From Motion, MicMac, Open-MVG, Web-service, Remote-computing

\begin{abstract}
:
Image based-modeling practices in the field of Cultural Heritage studies are nowadays no longer seen as one-shot applications but as various and complex multimodal scenarios. Current use of SFM and photogrammetric methods implies their extensions to facilitate the management of complex multimodal data sets carried-out by different experts around a single heritage asset. In order to fully benefit of collaborative semantic enrichment of spatially oriented resources, a versatile and robust solution have been developed to enable incremental registration of image-sets within the web-based platform AIOLI. For this purpose, this paper will present an on-going development of a Totally Automated Co-registration and Orientations (TACO) work-flow.
\end{abstract}

\section{INTRODUCTION}

Image-based modeling (IBM) state nowadays as the most used technique in $\mathrm{CH}$ field thanks to its versatility and lower cost compared to Range-based modeling. Nevertheless it requires higher technical skill in data capture process while the data processing is still divided between the complexity of open-source packages and the opacity of commercial black-box solutions. In this context the development on an open-source based automated pipeline seems a relevant choice for the scientific purposes of $\mathrm{CH}$ community. However, the automation is never an easy and straightforward task moreover when an incremental processing is required. Our solution to reach a Totally Automated Co-registration and Orientation of image sets (TACO) is a generic methodological approach of incremental and inter-operative photogrammetric processing. Integrated as the spatial registration core engine, it has been develop to allow the 2D/3D propagation of semantic annotations among multiples resources within AIOLI collaborative web-platform. It has been conceived as a flexible and evolutive pipeline so as to support a global data fusion methodology aiming to merge complex multimodal acquisitions (i.e. multisensor, multi-scalar, multi-spectral and/or simultaneously multitemporal). This article present the first implementation for automated processing of $\mathrm{CH}$ imaging multimodal acquisitions based on previous photogrammetric fusion experiments (Pamart et al., 2016, Pamart et al., 2017).

\subsection{Related Works}

As stated in a recent review article, the diversity of $\mathrm{CH}$ objects increases the complexity of defining and automating a efficient data fusion for $\mathrm{CH}$ purposes (Ramos and Remondino, 2015), especially when it requires the co-registration of $2 \mathrm{D}$ and $3 \mathrm{D}$ data. The overall process has been facilitated in our case restricted on IBM and dedicated to photographic-based acquisition (e.g. photogrammetry, technical photography and computational photography). Several related works achieved to link or bridge imaging techniques by exploiting feature-based registration method combined with photogrammetric spatialization, in multi-band (Barazzetti et al., 2010, Nocerino et al., 2018, Grifoni et al., 2018), multi-source (Chane et al., 2013, Yeh et al., 2016, Van der Perre et al., 2016) and/or multi-scale (Suwardhi et al., 2015) approaches. Whether if most of them are semi-automatic reproducible routines, as they remain isolated and case-studies dependant experiments, they are neither accessible to end-users nor aiming to cover the complete range of imaging modalities. Since the early stage of digital photogrammetry, remote-computing scenarios have been explored facing with robustness and versatility issues (Grussenmeyer et al., 2002, Nilsson et al., 2007, Heller et al., 2015, Alliez et al., 2017, Nocerino et al., 2017). Lately, the development and the deployment have been facilitating with the automation of both SFM and photogrammetric routines, including open-source packages (Moulon et al., n.d., Rupnik et al., 2017). However, after decades of development the relationship between SfM and photogrammetry remains fuzzy (Hartley and Mundy, 1993) while a combined used of respective tools emerge as an optimal solution (Aicardi et al., 2018). In order to fully benefit of the strength of each methods, libraries and algorithms, a need for inter-operative process is raising, moreover in the $\mathrm{CH}$ community where commercial softwares are progressively questioned in regard of necessity of preserving a data-provenance and dataquality continuum. A first attempt have been recently made in this direction to release a user-friendly tool (Gonzalez-Aguilera et al., 2018). With the on-going implementation and experimentation supported by TACO within AIOLI cloud service, our contribution is to make accessible an automated, versatile and robust pipeline for incremental photogrammetric processing.

\subsection{Aioli framework}

AIOLI is a reality-based 3D annotation platform designed for a multidisciplinary $\mathrm{CH}$ community to construct semantically enriched 3D descriptions of heritage assets starting from photogrammetric-friendly image set and spatialized annotations coupled with additional resources. This platform aims to impulse an innovative framework for the massive and large-scale collaborative $\mathrm{CH}$ documentation by linking features like image-based 3D reconstruction, 2D-3D spreading and correlation of semantic annotations, multi-layered analysis of qualitative and quantitative attributes. The service is deployed through a cloud-like infrastructure to make processing, visualization and annotation processes simply accessible via multi-device responsive web interfaces (PCs, tablets and smartphones) on-line and on-site. 


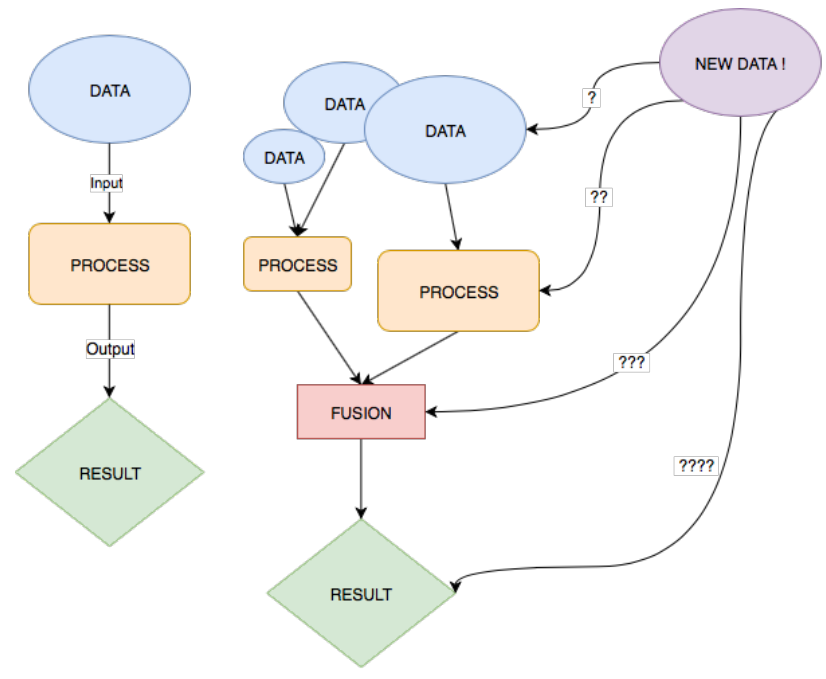

Figure 1: Current state and problematic of manual multimodal CRP data processing

For more information on updates or beta-testing program, visit the website (http:www.aioli.cloud/). Once logged into the workspace, AIOLI users can create a project linked to an Heritage Asset an is invited to upload an image set to obtain a first fully automatic photogrammetric reconstruction, powered by TACO. One of the main challenge is therefore to adapt and optimize the processing strategy for unknown input so as to increase the robustness that will hopefully lead to an accurate result of this first iteration (detailed below in section 2.2). Users can therefore start semantic annotation tasks on oriented pictures while their automatic 2D/3D re-projection within the scene is achieved in background (Manuel et al., 2016). However, photogrammetric-based documentation is no longer a linear work-flow, nor one shot application, neither a punctual work done by a single end-user, with the same equipment, experience and purposes. In order to face the pre-requisite need of collaborative documentation of $\mathrm{CH} \mathrm{ob-}$ jects, we developed incremental processing mode within TACO willing to support the complexity of multimodal acquisitions scenarios currently performed in $\mathrm{CH}$ domain. Therefore the possibility to add and merge new image-set through several iterations have been implemented according to the methodology and requirements of AIOLI's annotation framework. The complete description of the current stage of our versatile spatial registration method is detailed in section 2.3.

\section{TOTALLY AUTOMATED CO-REGISTRATION AND ORIENTATIONS}

The development of an IBM documentation solution can rely on the strengths of those techniques as stated in the literature (Remondino and El-Hakim, 2006) but can be criticized as well by its relative uncertainty - as an indirect measurement technique mostly related to quality of data given in input. In a conventional scenario the data processing is carried-out by the same expert or institution, in charge and responsible of the well-form and overall quality of data acquisition. This change of paradigm in a remotecomputing scenario implies two major changes. First, as the data given in input is user-dependant, it is presumed to be at least controlled if not optimal. Second, considering the unknowns of the input data (quality, size, acquisition strategy and purposes) an automated processing must be driven by compromising pragmatically between versatility, robustness and overall accuracy. Our approach prioritize accuracy starting by demanding tuning con-

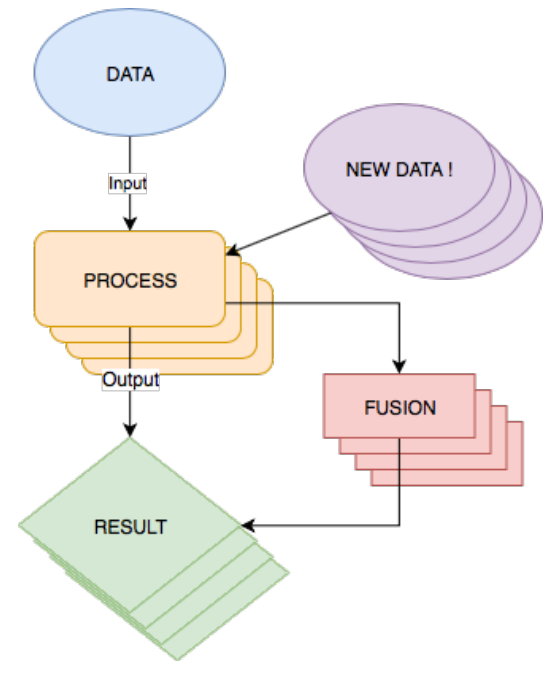

Figure 2: Proposed approach of automated incremental processing powered by TACO for the $\mathrm{CH}$ dedicated tool AIOLI

straints, progressively released to gain in robustness (and proportionally increasing uncertainty) with rebellious or sub-optimal dataset, the main drawback being the time of computation (compensated with our computation server capacities).

\subsection{Overview of a fully automatic and inter-operative 2D/3D registration pipeline}

An inter-operative process have been elaborated to benefit of the velocity and the accuracy of different open-source packages.

TACO aim to combines the latest improvements of Structure from Motion algorithms and the accuracy of Photogrammetric methods. The velocity and efficiency of Open-MVG (Moulon et al., n.d.) have been chosen for features extraction and 2D image matching steps, while its integration to AliceVision package opens new possibilities. The core engine of the spatial referencing of photographs and the $3 \mathrm{D}$ reconstruction is operated by the reliability and versatility of MicMac, developed mainly at IGN since 2003 (Rupnik et al., 2017) which has the benefit to have been evaluated in a metrological context contrary to some other solutions currently available (Toschi et al., 2014). The improvements in terms of automation and robustness achieved in the abovementioned packages facilitated the development and the optimization of ad-hoc processing pipelines as a mandatory target for the remote-computing scenario. TACO has been conceived to evolve with further improvement and development of solutions at the state of the art. Moreover it could be modulated according to dedicated pipelines and related specificity of multimodal data set requirements, as the RTI fusion framework recently presented (Pamart et al., 2019). Our workflow can therefore be levelled to the robustness of other current solutions from the open-source or commercial world, upgraded with following features :

a) Automatic best sub-sampling parametrization for tie-points extraction and matching using best-neighbours pre-selection

b) Self-adaptive incremental internal and external calibration

c) Automatic scaling and user defined world-space orientation based on QR-code detection (see Fig 4)

d) Fully automatic 3D dense matching

It has been formalized in a generic iterative/recursive script (represented in the diagram of the figure 3), taking image sets uploaded by users to define its optimized strategy and tuning of processing so as to ensure the best compromise in between accuracy and computation time. 


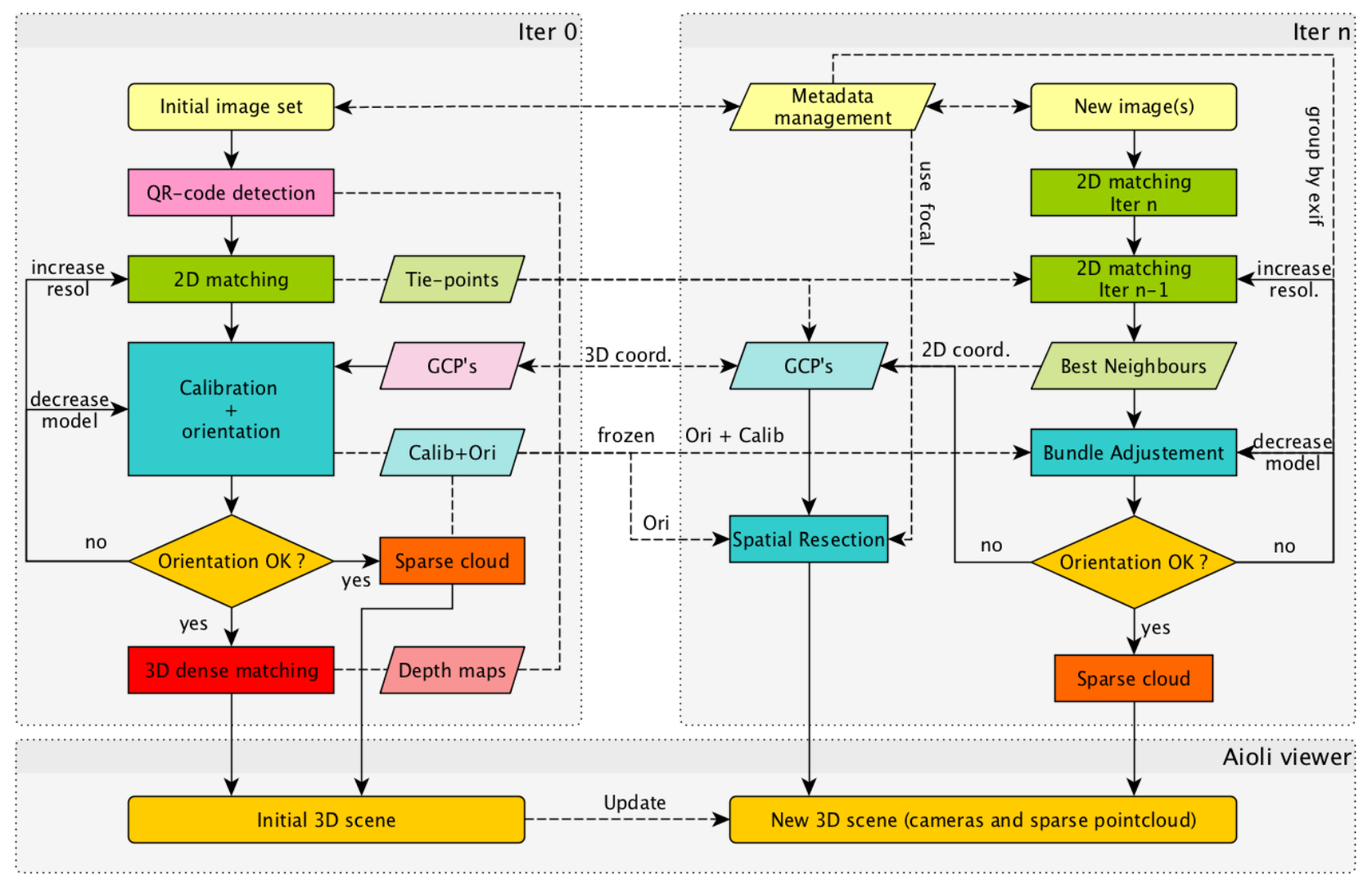

Figure 3: Diagram of the iterative co-registration processing for SO-RTI scene

\subsection{A robust initial iteration set as master acquisition}

The pictures of the first upload, assumed controlled and complete, are defined as a master acquisition meaning that the camera positions and resulting point cloud is (at this stage of development) frozen. The methodology of processing adopted is to initialize the computation with the highest degree of exigency and incrementally compromising on key parameters to enhance the robustness of this first iteration.

First, a special care is given to meta-data checking to i) verify their conformity and provide feedback to users ii) adapt the (re)processing strategy by EXIF grouping iii) anticipate data management among multiples image-sets that will be used to describe a heritage site or object.

Then, known as photogrammetric key feature, an iterative multiresolution approach aiming to find the appropriate range of homologous points, have been implemented to optimize further calibration and poses estimation steps. It is starting with a low resolution intended to speed-up the computation and the subsampling is recursively increased to medium then high resolution until a minimum of tie-points enabling an image alignment converging below an average residual threshold is not successfully completed. Additionally image matching ratio (Lowe, 2004) is increased and/or local feature enhancement (Rosu et al., 2015) is activated if images set are very rebellious (e.g. texture less or low-overlapping). Using this methodology help to ensure accuracy and robustness in the next alignment step. The latter efficiently combines the velocity of SfM method (based on trifocal tensor) to estimate external calibration and/or the reliability of standard photogrammetry method (based on bundle block adjustment and self-calibration) to compute internal calibration and refine camera poses. Until the condition of global orientation of all pictures is satisfied, the calibration model and polynomial degree of intrinsic parameters is progressively decreased, else an other image matching sub-sampling or strategy is required. Then an automatic shift from relative to absolute space coordinates (i.e. operating similarly to coded target is applied to scale and orient the model consistently to a user defined world-space, if QR-code have been recognized in the scene. Finally, a medium density point cloud (i.e. $1 \mathrm{pt}$ for $16 \mathrm{px}$ ) is generated by the performing and demanding multi-view stereo 3D dense matching algorithm of Micmac.

Therefore, this first iteration of the pipeline allows spatialising a first image set acquired by following a typical photogrammetric protocol. It is serving as initial geometric reference for adding new images during next iterations.

\subsection{A versatile incremental spatial registration of new im- ages on an existing image set}

The collaborative and multidisciplinary framework of AIOLI, has set the incremental registration of images as a priority. Moreover, the peculiarity of $\mathrm{CH}$ application domain and studies require to face with the challenging issue of the variety of multimodal scenari. The main difficulty to deal with, is the unknown overlapping gap between the image sets sequentially uploaded while they could have an high deviation in spatial or pixel resolution, radiometric consistency or changes in the scene or the object it-self.

Finding the best strategy and resolution to enhance features extraction and matching is therefore the essential step. If more than one image are added, they are first matched together before being matched with the rest of the dataset from previous iterations. As for the initial iteration, a multi-resolutionnal approach is included with the difference that the increment of the sub-sampling will re-use a best-neighbours selection to speed-up computation and discard bad or false key-points. 

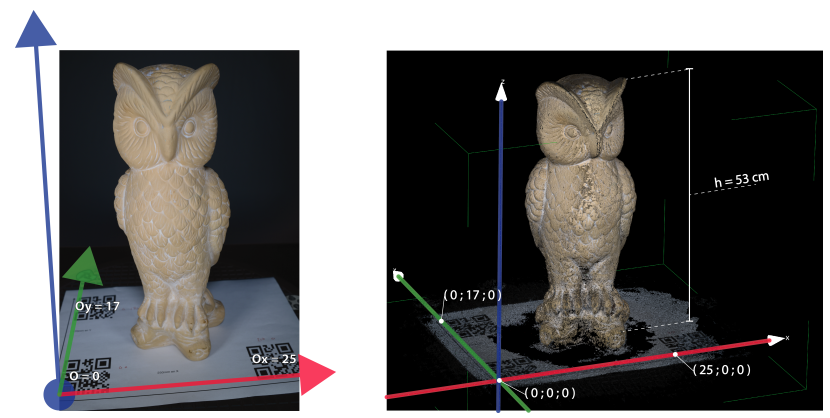

Figure 4: Example of QR-code based global orientation and scaling on small artefact dataset

Then, two alternative methods of spatial registration are integrated in the pipeline, both initialized from the results of the previous iterations (i.e. key-points, internal and external calibration, the dense point cloud) by fixing camera poses. A first Bundle Block Adjustment (BBA) based approach is applied, if a conventional photogrammetric dataset - understood a sequence of overlapping images - or even a single picture, is given in input. However according the deviation of resolution (image size, focal length or distance to the object), radiometry (exposure, spectral band, illumination) or context (parasites, alterations) BBA could be sub-optimal or even fail because of a lack of matched features (usually below 1000). A second surrogated approach is based on a strictly Spatial Resection approach (Shih and Faig, 1988) to calculate the pose of single camera (or several processed sequentially), which has also the benefit of being robust to missing meta-data (peculiar sensor, archive, analogic photography). This alternative is based on 11 parameters Direct Linear Transformation combined with RANSAC filtering (to remove outliers), to estimate both internal and external calibration among a known set of camera poses. As no Ground Control Points are available, we use the tie-points as 2D coordinates and their projection from depth-maps to retrieve corresponding 3D coordinates. This SR method is approximative but succeed to find an homographic relation with only tens points when BBA is delicate or impossible.

For BBA and SR approach as well the global accuracy and correctness is not related only to number of keypoints used for resection but more their redundancy and spatial distribution. Obviously, fewer are the key-points and their multiplicity, higher will be the uncertainty of the calibration and orientation computed with SR. Moreover, with both methods a result can converge to a false or odd solution for which some refinement methods will be prospected in a near future, like the incremental approaches of Open-MVG (Moulon et al., 2016, Ke and Roumeliotis, 2017), up-to-date perspective-three-point solver (Larsson et al., 2017, Persson and Nordberg, 2018) or using 2D/3D MutualInformation registration (Palma et al., 2010). However despite of current limitation, the outcome is encouraging for forthcoming extensions and modules of TACO to endorse its ability to process complex multimodal $\mathrm{CH}$ imaging data sets. Overall results in term of quality, accuracy and versatility are discussed and illustrated in the section below.

\section{RESULTS AND DISCUSSIONS}

To crashtest taco, we gathered a bunch of photogrammetric projects from our laboratory archives to reprocess them all using TACO. This evaluation is composed of hundred data sets, from 5 up to 300 images, acquired different sensor size and resolution (from smart-phones to full-frame DSLR), with a wide range of focal length (from $12 \mathrm{~mm}$ to $105 \mathrm{~mm}$ ). The algorithm successfully completed $99 \%$ of this wide and uneven selection of image sets

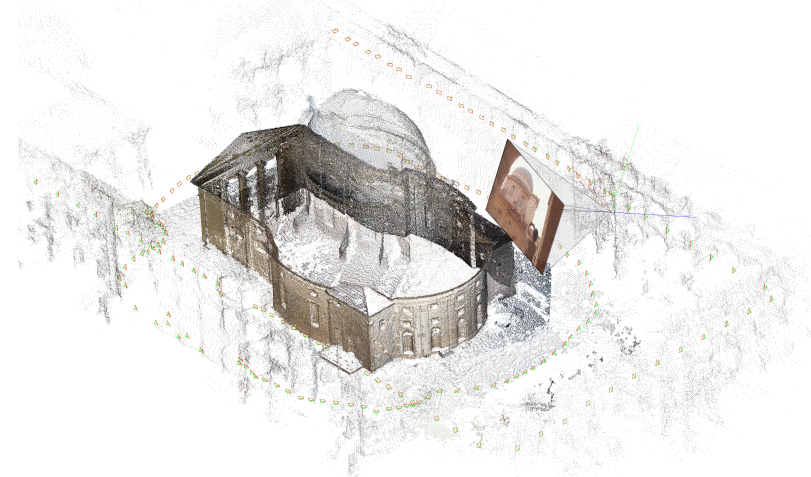

Figure 5: Isolated single picture registration using Spatial Resection on Building dataset

of various qualities, scales, complexities and typologies of $\mathrm{CH}$ objects, to a single condition ; images respect the basic prerequisites of photogrammetric capture. An extract, on pre-selected $\mathrm{CH}$ object typologies from this massive benchmark is given in table 1 for the initial iteration and the result of incremental processing is given in table 2 for which we tested the following multimodal scenari :

- The Facade dataset (Fig.7) proves TACO's ability to handle varied multi-focal acquisition

- The Portal dataset (Fig.8) shows TACO's robustness to integrate multi-temporal images including web-archive

- The Building dataset (Fig.5) illustrate TACO's velocity (40s of computing) register isolated picture on big image-set

- The Statue dataset (Fig.6) demonstrate TACO's versatility to manage complex UAV-based data-acquisition

- The Painting dataset (Fig.9) highlight TACO's capacity to deal with multi-scalar acquisition (14x GSD magnification)

The benchmark we present here have been processed directly on the AIOLI's computation server a Dell Power Edge R940, with a Intel Xeon CPU for a total of 144 cores of $3,0 \mathrm{GHz}, 256 \mathrm{~GB}$ of RAM and 4TB SSD storage. The pipeline is launched through Docker container (Merkel, 2014), thus computational times given (in table 1) for a single user exploiting full performance of the server may vary in case of simultaneous multi-users processing.

\begin{tabular}{|l||c||c|c|c|c|}
\hline Dataset & $\begin{array}{c}\text { Nb. } \\
\text { images }\end{array}$ & $\begin{array}{c}\text { Running } \\
\text { time }\end{array}$ & $\begin{array}{c}\text { Sparse } \\
\text { (k)points }\end{array}$ & $\begin{array}{c}\text { Dense } \\
(\mathrm{M}) \text { points }\end{array}$ & $\begin{array}{c}\text { ER2 } \\
(\mathrm{px})\end{array}$ \\
\hline Facade & 8 & $2 \mathrm{~min}$ & 102 & 2.35 & 1.08 \\
Fragment & 21 & $4 \mathrm{~min}$ & 60 & 1.34 & 0.47 \\
Portal & 26 & $7 \mathrm{~min}$ & 94 & 3.13 & 1.01 \\
Artefact & 40 & $7 \mathrm{~min}$ & 108 & 2.68 & 0.93 \\
Painting & 65 & $7 \mathrm{~min}$ & 127 & 1.76 & 0.74 \\
Excavation & 95 & $43 \mathrm{~min}$ & 641 & 1.34 & 0.49 \\
Temple & 134 & 120min & 1850 & 4.04 & 0.74 \\
Building & 181 & 170min & 224 & 1.14 & 0.73 \\
Statue & 273 & 390min & 1468 & 5.62 & 1.26 \\
\hline
\end{tabular}

Table 1: Composition and results of initial data sets extracted from the global benchmarking.

\begin{tabular}{|l||c||c|c|c|c|}
\hline Dataset & $\begin{array}{c}\text { Add. } \\
\text { images }\end{array}$ & $\begin{array}{c}\text { Nb. } \\
\text { Iter }\end{array}$ & $\begin{array}{c}\text { New } \\
\text { camera }\end{array}$ & $\begin{array}{c}\text { New } \\
\text { focal }\end{array}$ & $\begin{array}{c}\text { ER2 } \\
(\mathrm{px})\end{array}$ \\
\hline Facade & +107 & 12 & 1 & 6 & 1.54 \\
Portal & +4 & 4 & 4 & 4 & Nc. \\
Painting & +6 & 3 & 1 & 2 & 0.80 \\
Building & +1 & 1 & 1 & 1 & Nc. \\
Statue & +259 & 3 & 1 & 1 & 1.65 \\
\hline
\end{tabular}

Table 2: Details of multimodal dataset enrichment and results of incremental processing. 
The International Archives of the Photogrammetry, Remote Sensing and Spatial Information Sciences, Volume XLII-2/W9, 2019 8th Intl. Workshop 3D-ARCH "3D Virtual Reconstruction and Visualization of Complex Architectures", 6-8 February 2019, Bergamo, Italy

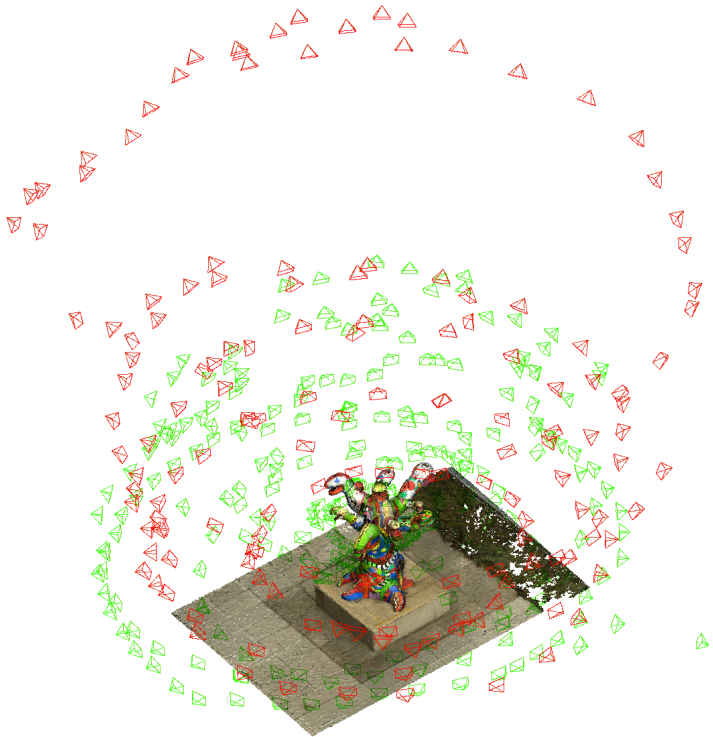

Figure 6: Fusion result of two UAV-based blocks of acquisition on Statue dataset

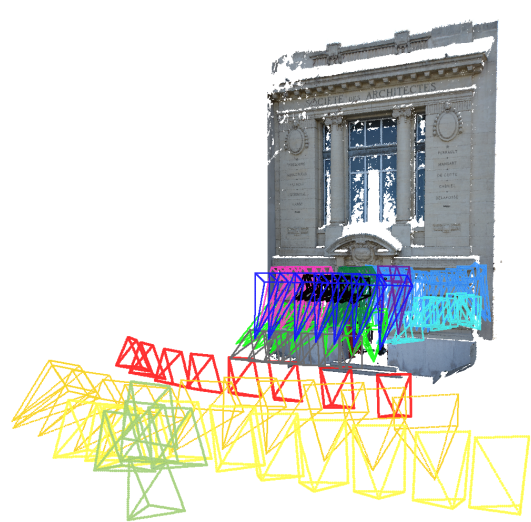

Figure 7: Example of complex multi-focal processing (each color represent an iteration) on Facade dataset

\section{CONCLUSION AND PERSPECTIVES}

The complementarity of BBA+SR approaches provides an efficient framework for incremental alignment of image sets and consequently offering a way to manage easily multi-modal acquisitions (including simultaneous multi-scale, multi-sensor, multitemporal or multi-spectral). Current stage of TACO is a generic formalization dedicated to AIOLI's integration, however it might evolve to treat more specifically peculiar acquisition modalities. The main condition for a TACO based fusion is thus related to the global correspondence and the photogrammetric consistency (i.e. spatial and resolution gaps, spectral or radiometric deviations, temporal or contextual variations) between the oriented pictures with the further new ones. Some improvements of processing steps or new features are currently planned, such as :

- Import of processed project, from MicMac, Open-MVG and PhotoScan

- Automatic bad or unconnected images removal

- Support of spherical images

- Integration of new descriptors like Maximal Self-Dissimilarity or Mutual Information to endorse radiometric robustness of image matching

- Extension of automatic GCP's extraction and coded targets support (Calvet et al., 2016)

- Automatic orthophotomosaic generation, included multiband support (Pamart et al., 2017)
The versatility and robustness of the iterative process allows currently the enrichment of the 3D scene by adding oriented pictures (even coming from a wide range of acquisition modalities). But at this stage of development, the spatially oriented resources are merged without generating or displacing other 3D coordinates to reduce internal process of AIOLI (computational costs of indexing and propagation steps). Nevertheless, the overall refinement of the camera positions as well as the point cloud through iterations (possible gain of accuracy, density or completion) required for the upcoming intreration of even more complex multimodal dataset - considering of the issues related to the management of resolutions and uncertainties variability - will be the focus of our future works and supported by :

- Metadata management to allow group by EXIF smart reprocessing

- Conversion to Alembic format (http://www.alembic.io/) to instantiate dynamic point-clouds among iterations

\section{ACKNOWLEDGEMENTS}

This work was partially supported by the ANR SUMUM project, grant Référence ANR-17-CE38-0004 of the French Agence Nationale de la Recherche, for which some of the case-studies presented in this paper as Painting and Statue correspond respectively to the Zett painting of Victor Vasarely from the Fondation Vasarely d'Aix-en-Provence and the Arbre-serpents sculpture of Niki de Saint Phalle from the Musée des Beaux-arts d'Angers.

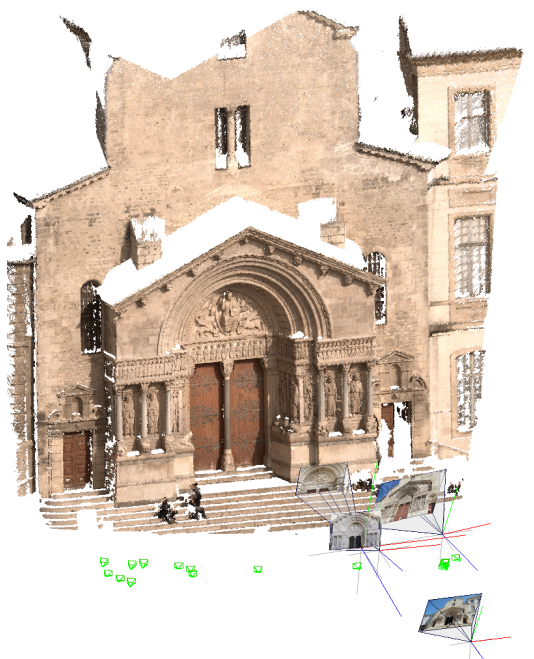

Figure 8: Spatial-orientation of web-based archives on Portal dataset

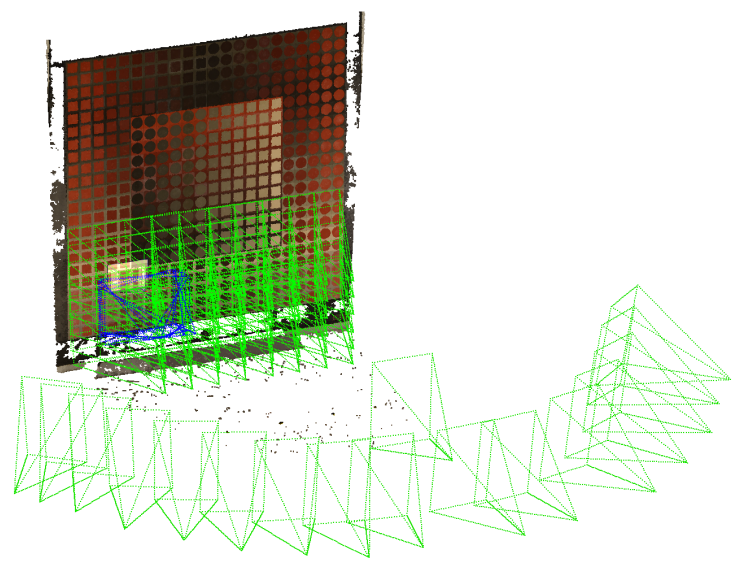

Figure 9: Data fusion with high scaling deviation Painting dataset 


\section{REFERENCES}

Aicardi, I., Chiabrando, F., Lingua, A. M. and Noardo, F., 2018. Recent trends in cultural heritage $3 \mathrm{~d}$ survey: The photogrammetric computer vision approach. Journal of Cultural Heritage.

Alliez, P., Forge, F., de Luca, L., Pierrot-Deseilligny, M. and Preda, M., 2017. Culture 3d cloud: A cloud computing platform for 3d scanning, documentation, preservation and dissemination of cultural heritage. ERCIM NEWS (111), pp. 35-35.

Barazzetti, L., Remondino, F., Scaioni, M., Lo Brutto, M., Rizzi, A. and Brumana, R., 2010. Geometric and radiometric analysis of paintings. International Archives of Photogrammetry, Remote Sensing and Spatial Information Sciences.

Calvet, L., Gurdjos, P., Griwodz, C. and Gasparini, S., 2016. Detection and accurate localization of circular fiducials under highly challenging conditions. In: Proceedings of the IEEE Conference on Computer Vision and Pattern Recognition, pp. 562-570.

Chane, C. S., Mansouri, A., Marzani, F. S. and Boochs, F., 2013. Integration of $3 \mathrm{~d}$ and multispectral data for cultural heritage applications: Survey and perspectives. Image and Vision Computing 31(1), pp. 91-102.

Gonzalez-Aguilera, D., López-Fernández, L., RodriguezGonzalvez, P., Hernandez-Lopez, D., Guerrero, D., Remondino, F., Menna, F., Nocerino, E., Toschi, I., Ballabeni, A. et al., 2018. Graphos-open-source software for photogrammetric applications. The Photogrammetric Record 33(161), pp. 11-29.

Grifoni, E., Legnaioli, S., Nieri, P., Campanella, B., Lorenzetti, G., Pagnotta, S., Poggialini, F. and Palleschi, V., 2018. Construction and comparison of $3 \mathrm{~d}$ multi-source multi-band models for cultural heritage applications. Journal of Cultural Heritage.

Grussenmeyer, P., Drap, P. and Gaillard, G., 2002. Arpenteur 3.0: Recent developments in web based photogrammetry. In: ARPENTEUR 3.0: Recent developments in web based photogrammetryISPRS, Commission VI: Education and Communications., pp. 1-7.

Hartley, R. I. and Mundy, J. L., 1993. Relationship between photogrammmetry and computer vision. In: Integrating photogrammetric techniques with scene analysis and machine vision, Vol. 1944, International Society for Optics and Photonics, pp. 92-106.

Heller, J., Havlena, M., Jancosek, M., Torii, A. and Pajdla, T., 2015. 3d reconstruction from photographs by $\mathrm{cmp}$ sfm web service. In: Machine Vision Applications (MVA), 2015 14th IAPR International Conference on, IEEE, pp. 30-34.

Ke, T. and Roumeliotis, S. I., 2017. An efficient algebraic solution to the perspective-three-point problem. arXiv preprint arXiv:1701.08237.

Larsson, V., Kukelova, Z. and Zheng, Y., 2017. Making minimal solvers for absolute pose estimation compact and robust. In: 2017 IEEE International Conference on Computer Vision (ICCV), IEEE, pp. 2335-2343.

Lowe, D. G., 2004. Distinctive image features from scaleinvariant keypoints. International journal of computer vision 60(2), pp. 91-110.

Manuel, A., Véron, P. and De Luca, L., 2016. 2d/3d semantic annotation of spatialized images for the documentation and analysis of cultural heritage. In: Proceedings of the 14th Eurographics Workshop on Graphics and Cultural Heritage, Eurographics Association, pp. 101-104.

Merkel, D., 2014. Docker: Lightweight linux containers for consistent development and deployment. Linux J.
Moulon, P., Monasse, P., Marlet, R. and Others, n.d. OpenMVG. An Open Multiple View Geometry library.

Moulon, P., Monasse, P., Perrot, R. and Marlet, R., 2016. Openmvg: Open multiple view geometry. In: International Workshop on Reproducible Research in Pattern Recognition, Springer, pp. $60-74$

Nilsson, D., Pletinckx, D., Van Gool, L. and Vergauwen, M., 2007. The arc $3 d$ webservice. EPOCH Knowhow book, available at http://www. her-it-age. net/, ISBN pp. 978-91.

Nocerino, E., Poiesi, F., Locher, A., Tefera, Y. T., Remondino, F., Chippendale, P. and Van Gool, L., 2017. 3d reconstruction with a collaborative approach based on smartphones and a cloudbased server. In: International archives of photogrammetry, remote sensing and spatial information sciences: Proceedings of the ISPRS Commission V Mid-Term Symposium'Close Range Image Measurement Techniques', Vol. 42number W8, Copernicus, pp. 187-194.

Nocerino, E., Rieke-Zapp, D., Trinkl, E., Rosenbauer, R., Farella, E., Morabito, D. and Remondino, F., 2018. Mapping vis and uvl imagery on $3 \mathrm{~d}$ geometry for non-invasive, non-contact analysis of a vase. International Archives of the Photogrammetry, Remote Sensing \& Spatial Information Sciences.

Palma, G., Corsini, M., Dellepiane, M. and Scopigno, R., 2010. Improving $2 \mathrm{~d}-3 \mathrm{~d}$ registration by mutual information using gradient maps. In: Eurographics Italian Chapter Conference, pp. 8994

Pamart, A., Guillon, O., Faraci, S., Gattet, E., Genevois, M., Vallet, J.-M. and De Luca, L., 2017. Multispectral photogrammetric data acquisition and processing for wall paintings studies. In: ISPRS-International Archives of the Photogrammetry, Remote Sensing and Spatial Information Sciences, Vol. 42, Copernicus GmbH, pp. 559-566.

Pamart, A., Guillon, O., Vallet, J.-M. and De Luca, L., 2016. Toward a multimodal photogrammetric acquisition and processing methodology for monitoring conservation and restoration studies. In: Proceedings of the 14th Eurographics Workshop on Graphics and Cultural Heritage, Eurographics Association, pp. 207-210.

Pamart, A., Ponchio, F., Abergel, V., Alaoui M'Darhri, A., Corsini, M., Dellepiane, M., Morlet, F., , Scopigno, R. and De Luca, L., 2019. A complete framework operating spatiallyoriented $\mathrm{rti}$ in a $3 \mathrm{~d} / 2 \mathrm{~d}$ cultural heritage documentation and analysis tool. ISPRS - International Archives of the Photogrammetry, Remote Sensing and Spatial Information Sciences XLII-2/W9, pp. in press.

Persson, M. and Nordberg, K., 2018. Lambda twist: An accurate fast robust perspective three point (p3p) solver. In: Proceedings of the European Conference on Computer Vision (ECCV), pp. 318-332.

Ramos, M. M. and Remondino, F., 2015. Data fusion in cultural heritage a review. ISPRS- Int. Archives of the Photogrammetry, Remote Sensing and Spatial Information Sciences 5, pp. W7.

Remondino, F. and El-Hakim, S., 2006. Image-based 3d modelling: A review. 21(115), pp. 269-291.

Rosu, A.-M., Assenbaum, M., De la Torre, Y. and PierrotDeseilligny, M., 2015. Coastal digital surface model on low contrast images. The International Archives of Photogrammetry, Remote Sensing and Spatial Information Sciences 40(3), pp. 307.

Rupnik, E., Daakir, M. and Pierrot Deseilligny, M., 2017. Mic$\mathrm{Mac}-\mathrm{a}$ free, open-source solution for photogrammetry. 2(1), pp. 14. 
Shih, T. and Faig, W., 1988. A solution for space resection in closed form. 27, pp. 547-556.

Suwardhi, D., Menna, F., Remondino, F., Hanke, K. and Akmalia, R., 2015. Digital 3d borobudur-integration of 3d surveying and modeling techniques. The International Archives of Photogrammetry, Remote Sensing and Spatial Information Sciences 40(5), pp. 417.

Toschi, I., Capra, A., De Luca, L., Beraldin, J.-A. and Cournoyer, L., 2014. On the evaluation of photogrammetric methods for dense $3 \mathrm{~d}$ surface reconstruction in a metrological context. II-5, pp. 371-378.

Van der Perre, A., Hameeuw, H., Boschloos, V., Delvaux, L., Proesmans, M., Vandermeulen, B., Van Gool, L. and Watteeuw, L., 2016. Towards a combined use of ir, uv and 3d-imaging for the study of small inscribed and illuminated artefacts. In: Lights On... Cultural Heritage and Museums!, FLUP, University of Porto, pp. 163-192.

Yeh, C.-K., Matsuda, N., Huang, X., Li, F., Walton, M. and Cossairt, O., 2016. A streamlined photometric stereo framework for cultural heritage. In: European Conference on Computer Vision, Springer, pp. 738-752. 